\title{
INHALT DES ZWEITEN BANDES
}

\section{Abschnitt: Schadensversicherung}

1. Titel: Vorschriften für die gesamte Schadensversicherung . . . . . . . . . . . . . . . 1

Vorbemerkungen zu $\S \S 49-80 \ldots \ldots \ldots \ldots \ldots$

I. Inhalt des Vertrages

$\S 49$ Ersatzleistung des Versicherers. Interesse. Kausalität. Bewirkung der Leistung . . . . . 39

I. Gefahrtragung des Versicherers . . . . . . . . . . . . . . . . . 42

II. Geld- und Naturalersatz . . . . . . . . . . . . . . . . . . . . 44

III. Interesse und Schaden . . . . . . . . . . . . . . . . . . . 56

IV. Kausalität und Schaden . . . . . . . . . . . . . . . . . . . . . . . 141

V. Bewirkung der Leistung . . . . . . . . . . . . . . . . . . . . . . 163

$\S 50$ Versicherungssumme ... . . . . . . . . . . . . . . . . . 170

I. Wesen der Versicherungssumme . . . . . . . . . . . . . . . 170

II. Arten der Versicherungssumme . . . . . . . . . . . . . . . . . 172

III. Zustandekommen der Versicherungssumme . . . . . . . . . . . . . . . 177

IV. Änderung der Versicherungssumme . . . . . . . . . . . . . . . . . . . 178

V. Rechtsbedeutung der Versicherungssumme . . . . . . . . . . . . . 180

VI. Abdingbarkeit der Vorschrift . . . . . . . . . . . . . . . . . . . . . 184

$\S 51$ Uberversicherung . . . . . . . . . . . . . . . . . . . . . . . . . . 184

I. Begriff der Uberversicherung . . . . . . . . . . . . . . . . . 186

II. Arten der Uberversicherung . . . . . . . . . . . . . . . . . . . 193

III. Einfache Überversicherung . . . . . . . . . . . . . . . . . . . 195

IV. Betrügerische Überversicherung ................... 206

$\S 52$ Versicherungswert. Eigentümerinteresse . . . . . . . . . . . . . . . . . . 211

I. Wesen des Versicherungswertes . . . . . . . . . . . . . . . 213

II. Bestimmung des Versicherungswertes . . . . . . . . . . . . . . . . 226

III. Rechtsbedeutung des Versicherungswertes . . . . . . . . . . . . . . . . . . 239

IV. Einfluß von Versicherungswertänderungen . . . . . . . . . . . . . . . . 242

V. Eigentümerinteresseversicherung nach $\S 52 \ldots \ldots . \ldots . \ldots . \ldots 243$

VI. Abdingbarkeit des $\S 52 \ldots \ldots . \ldots . \ldots . \ldots . . \ldots 244$

$\S 53$ Gewinnversicherung. . . . . . . . . . . . . . . . . . . . . . . 245

I. Begriff des entgehenden Gewinns . . . . . . . . . . . . . . . . 246

II. Abgrenzung des Gewinninteresses . . . . . . . . . . . . . . . . . . . . . 247

III. Arten der Gewinnversicherung . . . . . . . . . . . . . . . . . 250

IV. Vereinbarung der Gewinnversicherung . . . . . . . . . . . . . . . . . 256

V. Gefahren in der Gewinnversicherung . . . . . . . . . . . . . . . . . . . 257

VI. Schäden in der Gewinnversicherung . . . . . . . . . . . . . . . . 259

VII. Rechtsbehandlung der Gewinnversicherung . . . . . . . . . . . . . . 262

VIII. Abdingbarkeit des $\S 53 \ldots \ldots$. . . . . . . . . . . . . . . . 266 
$\$ 54$ Inbegriffsversicherung . . . . . . . . . . . . . . . . . . . 267

I. Zweck der Vorschrift . . . . . . . . . . . . . . . . . . . . . 268

II. Anwendungsbereich der Vorschrift . . . . . . . . . . . . . . . . . . 268

III. Wesen des Inbegriffs . . . . . . . . . . . . . . . . . 270

IV. Umschreibung des Inbegriffs . . . . . . . . . . . . . . . . . . . . . . 274

V. Rechtsbehandlung der Inbegriffsversicherung . . . . . . . . . . . . 284

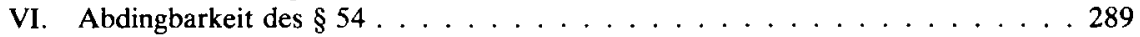

$\S 55$ Schaden. Bereicherungsverbot . . . . . . . . . . . . . . . . . . 290

I. Schadenshöhe als Leistungsbegrenzung . . . . . . . . . . . . . . . 291

II. Ableitung des Bereicherungsverbots . . . . . . . . . . . . . . . . . 292

III. Begriff des Schadens . . . . . . . . . . . . . . . . . . . . . 295

IV. Arten des Schadens . . . . . . . . . . . . . . . . . . . . . . . . . . . . 299

V. Versicherungsschaden im engeren und weiteren Sinn . . . . . . . . . 304

VI. Umfang des Schadens . . . . . . . . . . . . . . . . . . . . . 305

VII. Beweis des Schadens . . . . . . . . . . . . . . . . . . . . . . . . 313

VIII. Abwicklung des Schadens . . . . . . . . . . . . . . . . . . . . . . . 317

IX. Unabdingbarkeit des $\S 55 \ldots \ldots \ldots \ldots$. . . . . . . . . . . . . . . . . . .

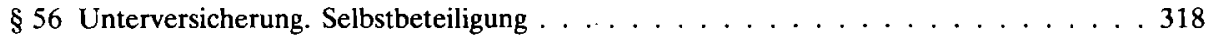

I. Übersicht . . . . . . . . . . . . . . . . . . . . . 320

II. Grundlagen der Unterversicherungsregelung . . . . . . . . . . . . . . 320

III. Begriff der Unterversicherung . . . . . . . . . . . . . . . . . 324

IV. Arten der Unterversicherung . . . . . . . . . . . . . . . . . 353

V. Rechtsfolgen der Unterversicherung . . . . . . . . . . . . . . 356

VI. Abdingbarkeit des $\$ 56 \ldots \ldots \ldots \ldots \ldots$. . . . . . . . . . . . . . . . . . . .

VII. Bekämpfung der Unterversicherung . . . . . . . . . . . . . . . . . . . . 363

VIII. Selbstbeteiligung des Versicherungsnehmers . . . . . . . . . . . . . 367

$\S 57$ Taxierte Versicherung . . . . . . . . . . . . . . . . . 380

I. Wesen $\operatorname{der}$ Taxe . . . . . . . . . . . . . . . . . . 381

II. Vereinbarung der Taxe . . . . . . . . . . . . . . . . . . . 389

III. Wirkungen der Taxe . . . . . . . . . . . . . . . . . . . 393

$\S 58$ Mehrfache und Mitversicherung . . . . . . . . . . . . . . . . . . . . . 409

I. Beteiligung mehrerer Versicherer . . . . . . . . . . . . . . . . 411

II. Speziell: Mehrfache Versicherung . . . . . . . . . . . . . . . 414

III. Speziell: Mitversicherung . . . . . . . . . . . . . . . . . . . . . . . . . . 443

$\S 59$ Doppelversicherung und Subsidiarität . . . . . . . . . . . . . . . . . . . 459

I. Begriff der Doppelversicherung . . . . . . . . . . . . . . . . 461

II. Arten der Doppelversicherung . . . . . . . . . . . . . . . . . . . 468

III. Rechtsbehandlung der Doppelversicherung . . . . . . . . . . . . . 468

IV. Subsidiarität von Versicherungen . . . . . . . . . . . . . . . . . 489

$\S 60$ Beseitigung der Doppelversicherung . . . . . . . . . . . . . . . . . . 497

I. Ubersicht über Beseitigungsrecht . . . . . . . . . . . . . . . . . . 498

II. Voraussetzungen des Beseitigungsrechts . . . . . . . . . . . . . . . . . . . 498

III. Verlangen der Beseitigung . . . . . . . . . . . . . . . . . . 504

IV. Rechtsfolgen des Verlangens . . . . . . . . . . . . . . . . . . . 509

V. Abdingbarkeit des Beseitigungsrechts . . . . . . . . . . . . . . 511

$\$ 61$ Herbeiführung des Versicherungsfalls . . . . . . . . . . . . . 512

I. Ubersicht . . . . . . . . . . . . . . . . . . . . 515

II. Anwendungsbereich .................. 516 


\section{Inhalt des zweiten Bandes}

III. Rechtsnatur . . . . . . . . . . . . . . . . . . . . . . . . . . . 524

IV. Abgrenzung . . . . . . . . . . . . . . . . . . . . . . . 529

V. Objektive Voraussetzungen . . . . . . . . . . . . . . . . 533

VI. Subjektive Voraussetzungen . . . . . . . . . . . . . . . . . . . . 544

VII. Einstehen für Dritte . . . . . . . . . . . . . . . . . . . . . . 572

VIII. Rechtsfolgen der Herbeiführung . . . . . . . . . . . . . . . . . . . . 595

IX. Abdingbarkeit des $\$ 61 \ldots \ldots \ldots \ldots \ldots$. . . . . . . . . . . . . . . . . . . . .

X. Ausstrahlungen des $\S 61 \ldots \ldots \ldots \ldots$. . . . . . . . . . . 609

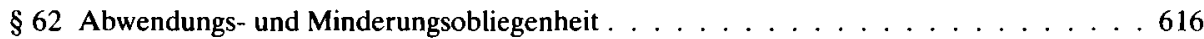

I. Ubersicht . . . . . . . . . . . . . . . . . . . . . . . . 617

II. Rechtsnatur . . . . . . . . . . . . . . . . . . . . . 620

III. Abgrenzung . . . . . . . . . . . . . . . . . . . . . . . . . . 622

IV. Objektiver Tatbestand . . . . . . . . . . . . . . . . 626

V. Subjektiver Tatbestand . . . . . . . . . . . . . . . . . . . . . . 643

VI. Rechtsfolgen der Verletzung . . . . . . . . . . . . . . . . . . . . 646

VII. Unabdingbarkeit der Vorschrift . . . . . . . . . . . . . . . . . . . . . . . 649

$\S 63$ Ersatz von Aufwendungen . . . . . . . . . . . . . . . 650

I. Ubersicht . . . . . . . . . . . . . . . . . . . .6651

II. Rechtsnatur . . . . . . . . . . . . . . . . . . . . 652

III. Begriff der Aufwendung . . . . . . . . . . . . . . . . . . 653

IV. Arten der Aufwendung . . . . . . . . . . . . . . . . . 655

V. Zweckrichtung der Aufwendung . . . . . . . . . . . . . . . . . 659

VI. Erforderlichkeit der Aufwendung . . . . . . . . . . . . . . . . . . . . 661

VII. Anspruch auf Aufwendungsersatz . . . . . . . . . . . . . . . . 662

VIII. Abdingbarkeit des $\S 63 \ldots \ldots$. . . . . . . . . . . . . . . . . 668

IX. Konkurrierende Ansprüche . . . . . . . . . . . . . . . . . . . . . . 669

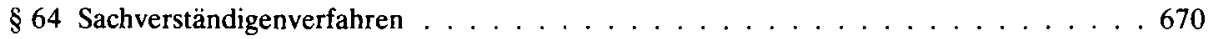

I. Verfahren der Schadensabwicklung . . . . . . . . . . . . . . . . . 671

II. Bedeutung des Sachverständigenverfahrens . . . . . . . . . . . . . . 672

III. Rechtsquellen des Sachverständigenverfahrens . . . . . . . . . . . . . . . . . 673

IV. Abgrenzung des Sachverständigenverfahrens . . . . . . . . . . . . . . . 675

V. Vereinbarung des Sachverständigenverfahrens . . . . . . . . . . . . . 678

VI. Der Sachverständige . . . . . . . . . . . . . . . . . . . . . . 683

VII. Das Verfahren . . . . . . . . . . . . . . . . . . . . . . . . 695

VIII. Die Feststellung . . . . . . . . . . . . . . . . . . . . 700/5

IX. Die Unabdingbarkeit . . . . . . . . . . . . . . . . . . 700/16

$\S 65$ Vertretung des Versicherungsnehmers . . . . . . . . . . . . 700/17

I. Grundsatz . . . . . . . . . . . . . . . . . . . . . . 700/17

II. Tragweite . . . . . . . . . . . . . . . . . . . . . . 700/17

III. Fallgruppen . . . . . . . . . . . . . . . . . . . . . 700/18

IV. Zwangscharakter . . . . . . . . . . . . . . . 700/20

$\S 66$ Schadensabwicklung. Ermittlungs- und Feststellungskosten . . . . . . . . . 700/21

I. Formen der Schadensabwicklung . . . . . . . . . . . . . . . . . . 700/21

II. Ermittlungs- und Feststellungskosten . . . . . . . . . . . . . . . . . . 700/34

III. Abdingbarkeit des $\S 66 \ldots \ldots \ldots \ldots$. . . . . . . . . . . . . 700/42

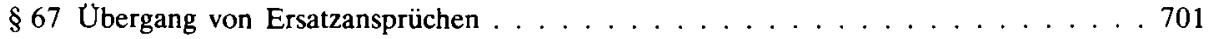

I. Grundlegung . . . . . . . . . . . . . . . . . . . . 706

II. Außenversicherungsrechtliche Voraussetzungen des Ubergangs . . . . . . . . . 716

III. Versicherungsrechtliche Voraussetzungen des Úbergangs . . . . . . . . . . . . . 729 
IV. Umfang und Sicherung des Ubergangs . . . . . . . . . . . . . . . 737

V. Wirkungen des Ubergangs . . . . . . . . . . . . . . . . . . . . 748

VI. Ausschluß des Úbergangs . . . . . . . . . . . . . . . . . . . . . . . . . . . . . . . . . . . . . . . . . . . . . .

VII. Mehrheit der Beteiligten . . . . . . . . . . . . . . 762

VIII. Verfahrensrecht . . . . . . . . . . . . . . . . . 770

IX. Geltungsbereich . . . . . . . . . . . . . . . . . . . . 774

X. Surrogatslösungen . . . . . . . . . . . . . . . . . . 786

Nachtrag . . . . . . . . . . . . . . . . . . . 791

$\S 68$ Interessemangel . . . . . . . . . . . . . . . . . . . . 793

I. Grundlegung . . . . . . . . . . . . . . . 796

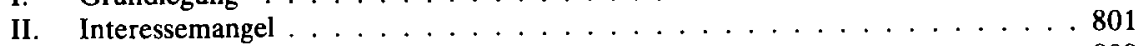

III. Anfänglicher Interessemangel . . . . . . . . . . . . . . . . . . . 809

IV. Nachträglicher Interessemangel (Interessewegfall; $\S 68$ II-IV) . . . . . . . 814

V. Versicherung für fremde Rechnung . . . . . . . . . . . . . . 818

VI. Partieller Interessemangel . . . . . . . . . . . . . . . . . . . 823

VII. Halbzwingender Charakter . . . . . . . . . . . . . . . 830

$\S 68$ a Unabdingbarkeit . . . . . . . . . . . . . . . . . . . . . . . . . . . . . . . .

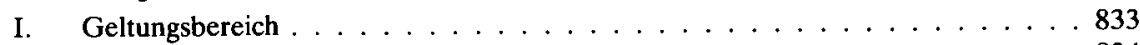

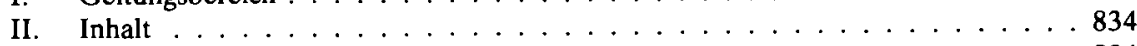

III. Die einzelnen halbzwingenden Vorschriften . . . . . . . . . . 834

II. Veräußerung der versicherten Sache

Vorbemerkungen zu $\$ 869-73 \ldots \ldots \ldots$. . . . . . . . . . . . . . 835

$\S 69$ Ubergang des Versicherungsverhältnisses . . . . . . . . . . . . . . . . . . 840

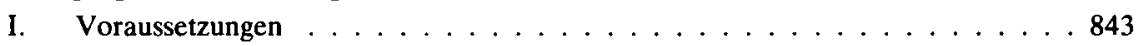

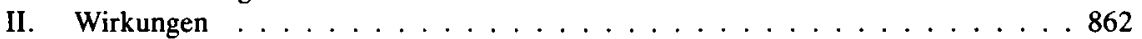

$\S 70$ Kündigungsrecht bei Veräußerung . . . . . . . . . . . . . . . . . . 874

I. Allgemeines zum Kündigungsrecht (Voraussetzungen und Einwendungen) . . . 876

II. Kündigungsrecht des Versicherers $(\S 70 \mathrm{I}) \ldots \ldots \ldots \ldots$. . . . . . . 883

III. Kündigungsrecht des Erwerbers $(\$ 70 \mathrm{II}) \ldots \ldots \ldots \ldots 8 . \ldots \ldots$

IV. Wirkungen der Kündigung (insbes. $\$ 70$ III) . . . . . . . . . . . . 889

V. Gesetzeskonkurrierende Vorschriften . . . . . . . . . . . . . . . . . 895

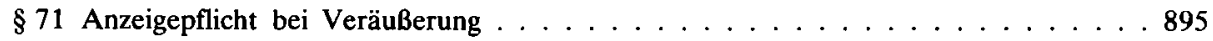

I. Zusammenhang mit $\$ 70 \ldots \ldots \ldots \ldots \ldots$. . . . . . . . . . . . . . . . . . .

II. Erfordernisse der Anzeige . . . . . . . . . . . . . . . . . . . . . 897

III. Rechtsnatur der Anzeigepflicht . . . . . . . . . . . . . . . . 900

IV. Wirkungen der Anzeige . . . . . . . . . . . . . . . . . . . 902

V. Wirkungen der Unterlassung der Anzeige . . . . . . . . . . . . . . . . 902

VI. Beweislast . . . . . . . . . . . . . . . . . . . . . . 906

VII. Konkurrenzen . . . . . . . . . . . . . . . . . . . . . 907

$\$ 72$ Unabdingbarkeit . . . . . . . . . . . . . . . . . . . . . . 909

I. Normativer Geltungsbereich . . . . . . . . . . . . . . . . . 909

II. Halbzwingende Natur . . . . . . . . . . . . . . . . . . . . . . . . 910

III. Bestimmungen im Versicherungsvertrag . . . . . . . . . . . . . . . 911

IV. Bestimmungen in sonstigen Vereinbarungen . . . . . . . . . . 914 
Inhalt des zweiten Bandes

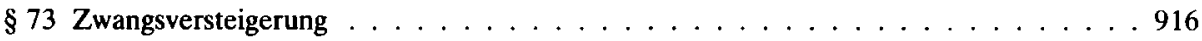

I. Bedeutung . . . . . . . . . . . . . . . . . . 916

II. Tatbestände . . . . . . . . . . . . . . . . . . . . 917

III. Durchführung . . . . . . . . . . . . . . . . . . . . . . . 918

Nachtrag Vorbem. zu $\$ \S 69-73 \ldots \ldots \ldots$. . . . . . . . . . . . . . . . . . .

III. Versicherung für fremde Rechnung

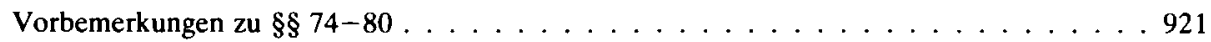

$\$ 74$ Begriff . . . . . . . . . . . . . . . . . . . . . . . . .927

I. Charakteristik der Versicherung für fremde Rechnung . . . . . . . . . . . 928

II. Beziehungen zwischen Versicherungsnehmer und versichertem Interesse . . . . 930

III. Versicherung für fremde Rechnung in der Lebensversicherung? . . . . . . . . . . 933

IV. Vertragsschlu $B \ldots \ldots \ldots \ldots \ldots . \ldots \ldots \ldots$

V. Anfechtbarkeit . . . . . . . . . . . . . . . . . . . . . . 939

VI. Beendigung des Versicherungsvertrages für fremde Rechnung . . . . . . . . . . 941

$\S 75,76$ Stellung des Beteiligten zum Versicherer . . . . . . . . . . . . . . . . 943

I. Uberblick . . . . . . . . . . . . . . . . . . . . . . . . . . . . 944

II. Vollzugsverhältnis . . . . . . . . . . . . . . . . . . . . 945

III. Deckungsverhältnis . . . . . . . . . . . . . . . . 963

$\S 77$ Rechtsverhältnis zwischen Versicherungsnehmer und Versichertem . . . . . . . . 964

1. Uberblick . . . . . . . . . . . . . . . . . . . . . . . . . . 965

II. Ansprüche des Versicherungsnehmers gegen den Versicherten bei intaktem Innen-

verhältnis . . . . . . . . . . . . . . . . . . . . . 966

III. Ansprüche des Versicherten gegen den Versicherungsnehmer . . . . . . . . . . . 973

IV. Ansprüche des Versicherungsnehmers gegen den Versicherten bei fehlendem Innenverhältnis . . . . . . . . . . . . . . . . . 978

$\S 78$ aufgehoben

$\S 79$ Kenntnis und Verhalten des Versicherten . . . . . . . . . . . . . . . . . . 979

I. Uberblick . . . . . . . . . . . . . . . . . . . . . 980

II. Tatbestandsmerkmale des $\$ 79$ I . . . . . . . . . . . . . . . . . 980

III. Tatbestandsmerkmale des $\$ 79$ II, III . . . . . . . . . . . . . . . . . 983

IV. Kombinierte Eigen- und Fremdversicherung . . . . . . . . . . . . . . . . 984

V. Besonderheiten bei der Personenversicherung . . . . . . . . . . . . . . 987

$\S 80$ Vermutung für Eigenversicherung; Versicherung für Rechnung „wen es angeht“ . . . 988

I. Charakteristik des $\$ 80 \mathrm{I} \ldots \ldots . \ldots . \ldots 989 . \ldots \ldots$

II. Deckung von Interessen an fremder Sache . . . . . . . . . . . . . . . 991

III. Versicherung für Rechnung wen es angeht $(\$ 80 \mathrm{II}) \ldots \ldots \ldots . \ldots . \ldots 92$

Sachregister . . . . . . . . . . . . . . . . . . . . . . 1007 
Auf das Verzeichnis der Abkürzungen in Band I S. XV-XVII wird verwiesen.

Das Schrifttum ist grundsätzlich bei den einzelnen Vorschriften angeführt. Für die $\S \S 69-73$ findet es sich in den Vorbemerkungen zu $\S \S 69-73$ Anm. 2, für die $\S \S 74-79$ in den Vorbemerkungen zu $\S \S 74-80$ Anm. 2. 